\title{
Investigation of Wireless Sensor Networks for Structural Health Monitoring
}

\author{
Ping Wang, ${ }^{1}$ Yan Yan, ${ }^{1}$ Gui Yun Tian, ${ }^{2}$ Omar Bouzid, ${ }^{2}$ and Zhiguo Ding ${ }^{2}$ \\ ${ }^{1}$ College of Automation Engineering, Nanjing University of Aeronautics and Astronautics, Yudaojie Road 29, \\ Jiangsu 210016, China \\ ${ }^{2}$ School of Electrical and Electronic Engineering, Newcastle University, Newcastle upon Tyne NE1 7RU, UK
}

Correspondence should be addressed to Ping Wang, zeit@263.net

Received 10 November 2011; Accepted 6 January 2012

Academic Editor: Raimond Grimberg

Copyright () 2012 Ping Wang et al. This is an open access article distributed under the Creative Commons Attribution License, which permits unrestricted use, distribution, and reproduction in any medium, provided the original work is properly cited.

\begin{abstract}
Wireless sensor networks (WSNs) are one of the most able technologies in the structural health monitoring (SHM) field. Through intelligent, self-organising means, the contents of this paper will test a variety of different objects and different working principles of sensor nodes connected into a network and integrated with data processing functions. In this paper the key issues of WSN applied in SHM are discussed, including the integration of different types of sensors with different operational modalities, sampling frequencies, issues of transmission bandwidth, real-time ability, and wireless transmitter frequency. Furthermore, the topology, data fusion, integration, energy saving, and self-powering nature of different systems will be investigated. In the FP7 project "Health Monitoring of Offshore Wind Farms," the above issues are explored.
\end{abstract}

\section{Background}

Wireless sensor networks (WSNs) are considered one of the most important technologies in the 21st century, which will have a profound impact on the future way of life for humankind. A typical wireless sensor network consists of wireless communication, data acquisition, processing, and fusion stages. The sensor nodes are self-organised through a specific protocol and are able to obtain information about the surrounding environment, working together to accomplish specific tasks. The technology spans many fields, such as wireless communication, network technology, integrated circuits, sensor technology, microelectromechanical systems (MEMS), and embedded systems, to name just a few. Figure 1(a) illustrates the typical basic architecture of a sensor network, and Figure 1(b) shows the typical hardware of a sensor network node. In February 2003, "Technology Review," a magazine published in the United States, selected ten far-reaching impacts of emerging technologies, where sensor networks were ranked in the first place. The US Department of Defense gave high priority to wireless sensor networks and put it as an important research area, establishing a series of military research projects. Intel, Microsoft, and other companies also have carried out some research in the field.

Not only industry and defense organisations have shown strong interest in wireless sensor networks, some world class universities such as UCLA, MIT, Cornell University, and the University of California, Berkeley [1] also have carried out research on WSN and achieved some results. The University of California, Berkeley, presented network connectivity reconstruction method for sensor location, based on the correlation of the data coding modes, to determine the location of sensor network nodes; they also developed a sensor operating system, TinyOS. The Massachusetts Institute of Technology studied sensor network data stream management systems integration frameworks, query optimisation, network congestion control for energy saving purposes, along with middleware technologies such as positioning, tracking, networking, and scalable algorithms for large-scaled sensor networks. These studies received NSF, DARPA, and the Air Force Space Laboratory support. The ZigBee Alliance and IEEE also developed standards of Chinese version IEEE 802.15.4c [2].

After developing further in the past several years, some applications of wireless sensor networks have been turned 


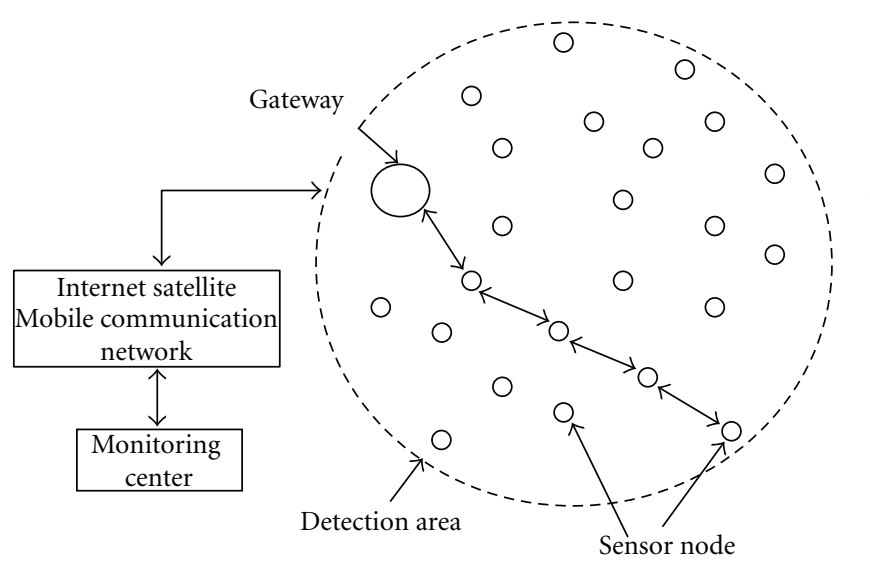

(a)

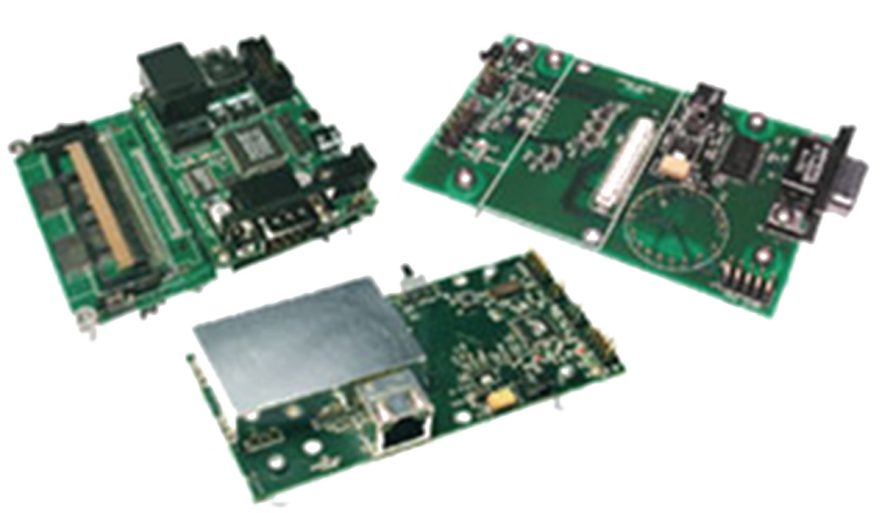

(b)

Figure 1: (a) a sensor network architecture. (b) hardware of sensor network nodes.

into products. Nowadays a number of WSN research platforms have been developed successfully, such as the University of California, Berkeley TinyOS/Mica, Smart Dust, and PicoRadio platforms. The ZigBee Alliance was codeveloped by Invensys, Mitsubishi Electric, TI, Motorola, Philips, and more than 20 other semiconductor and IT companies. Meanwhile, the IEEE has also developed the IEEE802.15.4 standards and has established a number of demonstration systems. Crossbow has developed a series of modules and products based on the ZigBee protocol and TinyOS, which can be used to form a WSN. TI has also developed some solutions based on ICs such as CC2431, which embed the ZigBee protocol of MAC and PHY on them and offer developments that contain the software and firmware of the complete ZigBee protocol stack [3].

Structural health monitoring (SHM) is a typical area amongst the many possible applications of wireless sensor networks. SHM is an emerging research area and is focussed on the field of infrastructure $[4,5]$, mainly on the integration and application of sensors, signal processing, and communication technologies. It also focuses on complex engineering systems and infrastructure to prevent structural failure and disaster, such as with the monitoring of bridges, large buildings, and dams. One of the possible SHM applications is that of a wind power monitoring system illustrated by the EU science and technology key project FP7 "Health Monitoring of Offshore Wind Farms." By 2020, China wind power capacity will reach 150 million kilowatts [6], and nondestructive testing (NDT) and SHM are useful means with which to reduce the maintenance costs of wind power, and to extend the lifetime of infrastructure, and to ensure the safety of power supply. As shown in Figure 2, the project aims to analyse wind power generation systems and to develop intelligent WSN and SHM technologies for the wind turbine blade, gearbox, generator, power electronics, and other structural components. In this project, a complete wind power system health analysis, life cycle assessment, fault diagnosis, maintenance management programs, planning and scheduling system of a complete windpower system is provided, including the design, production, installation, maintenance, and supply chain feedback.

In Section 2, the key problems acting against the implementation of WSNs for SHM on wind power systems will be identified and discussed.

\section{The Key Problems of Wireless Sensor Networks Combined with Structural Health Monitoring}

As briefly discussed in Section 1, the key problems to developing such an SHM system in conjunction with WSN are summarised as follows: the problem of compatibility between different sensors, their sampling frequencies, and operational modes, the problem of transmission bandwidth and real-time ability variance, the selection of a wireless transmission frequency, topology choice, data fusion method, and the contrast between the energy consumption requirements of different applications to that of each different device. In the following subsections, each individual problem will be discussed in detail.

2.1. Sampling Frequency and Operation Mode. In the field of structural health monitoring, various types of sensors are used, including, but not restricted to, resistance strain, piezoelectric vibration, optical fiber strain, dip angle, acoustic emission, and stress measurement sensors [7]. Each of these sensors has different physical mechanisms and should thus be operated in different ways; some examples are given as follows.

(1) The signals of strain, deformations, and dip angle are static or of low frequency, and they usually work at low sampling frequencies. For example, some of the signals in a strain test are sampled by a frequency lower than $1 \mathrm{~Hz}$. The likelihood of a request for data processing and real-time transmission is low.

(2) Vibration sensors usually measure objects where the level of vibration can range from dozens of $\mathrm{Hz}$ to 


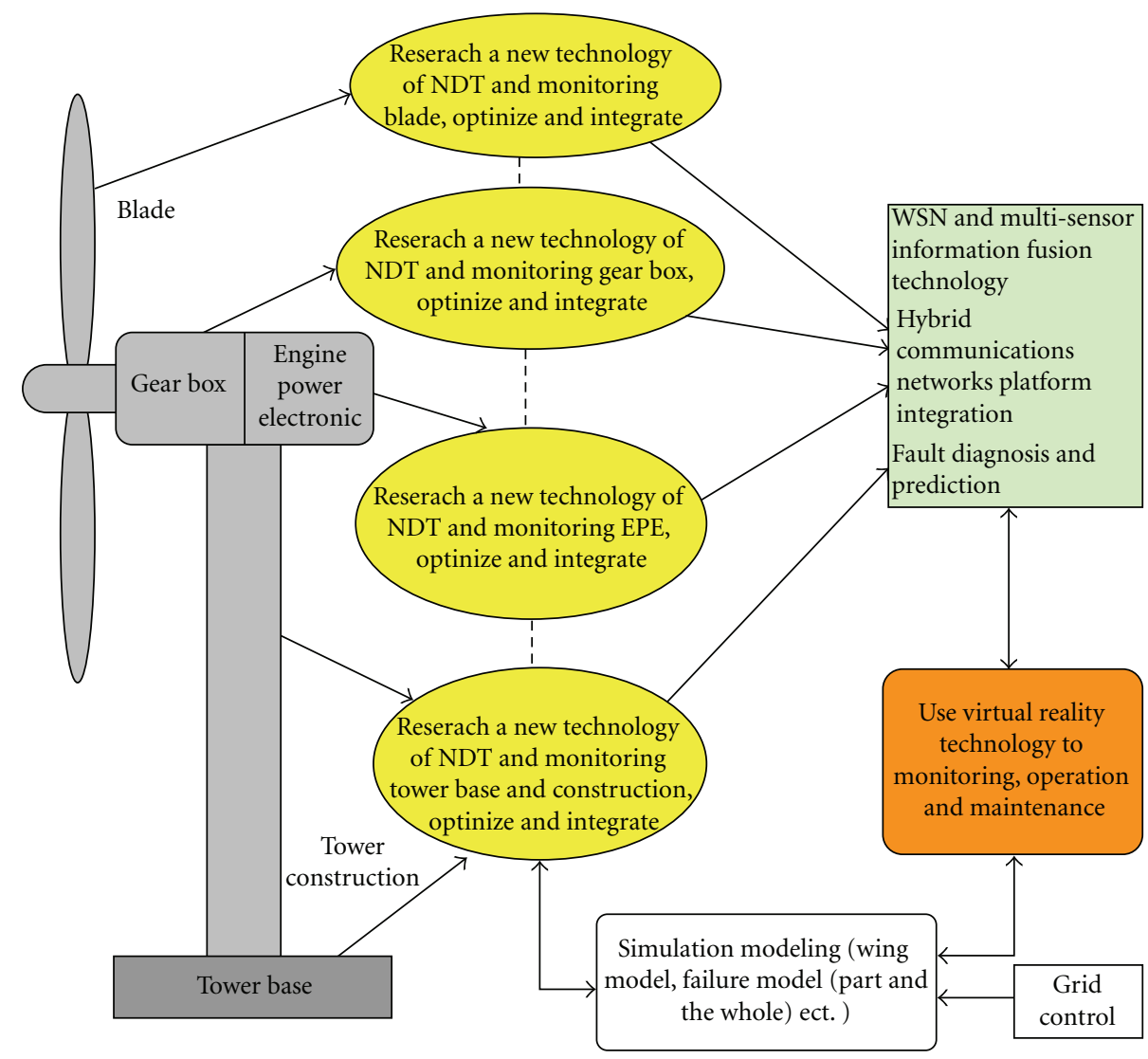

Figure 2: The system architecture of FP7 project "Health Monitoring of Offshore Wind Farms".

TABLE 1: The carrier frequency and transmission bandwidth of ZigBee Alliance and the IEEE 802.15.4 standard.

\begin{tabular}{lccc}
\hline Carrier frequency & Band nature & Maximum bandwidth & Frequency point \\
\hline $2.4 \mathrm{GHz}$ & ISM Worldwide & $250 \mathrm{kbps}$ & 16 \\
$868 \mathrm{MHz}$ & Europe & $20 \mathrm{kbps}$ & 1 \\
$915 \mathrm{MHz}$ & ISM Americas & $40 \mathrm{kbps}$ & 10 \\
$780 \mathrm{MHz}$ & $802.15 .4 \mathrm{c}$ (Chinese) & $250 \mathrm{kbps}$ & 8 \\
\hline
\end{tabular}

hundreds of $\mathrm{KHz}$. Thus, there is a much greater requirement from the system in terms of sampling frequency, data processing, and transmission. The added need for synchronisation from the system adds an extra burden.

(3) Eddy current, pulsed magnetic flux leakage, and other nondestructive testing sensors are used for the monitoring of key components, where signals are sampled with a frequency of more than a few hundred $\mathrm{Hz}$. In addition to this the requirement of data processing and analytical abilities from the WSN nodes is very high.

(4) The imaging sensors require the WSN nodes to have the ability to carry out high-speed data transmission. They also require WSN nodes to have certain decoding and image processing abilities.
As the task of structural health monitoring systems is a complicated one, consisting of the monitoring of many physical and electrical failures in different components, they need various sensors working together. The choices of the sensor network sampling frequency, from several $\mathrm{Hz}$ to several hundreds of $\mathrm{kHz}$, working mode, and compatibility must be considered when choosing each node.

\subsection{Transmission Bandwidth and Real-Time Monitoring. In} general, with the requirements of low cost and low power, the design of WSN has mainly been for low bandwidth and non/nonurgent real-time applications, as shown in Table 1. For applications such as SHM, designs based on lowbandwidth and non-real-time systems can only be applied to strain deformation or dip monitoring, which require only slowly varying signal sensing and transmission. The data from vibration measurements as well as those resulting from image acquisition require a higher transmission bandwidth. 


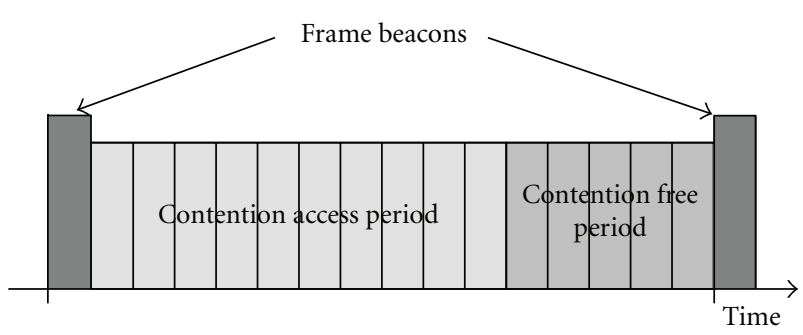

Figure 3: ZigBee MAC time slot with competition and sub-slot competition.

In addition to these, for some alarm transmission with high real-time requirements, such as those from pulsed eddy current measurement node (which can receive harbinger information of the sudden occurrence of crack), special considerations with regard to real-time network transmission need to be undertaken.

Except for the physical communication and processing capacity of a system, the protocols, especially MAC protocols, influence the real-time ability and transmission bandwidth of network communication $[8,9]$. MAC technology usually consists of two different mechanisms, competitive and noncompetitive mechanisms. The competitive MAC mechanism, carrier sense multiple access with collision avoidance (CSMA/CA), is most commonly used. Distributed layer MAC protocols, which are based on the CSMA/CA protocol, use a sleep cycle mechanism to limit energy consumption. But a larger network time delay is produced as the system needs to be awoken from a sleep cycle before the data can first be sent to it and then transmitted onwards. In addition, the T-MAC (MAC) protocol transmits all of the data through a variable-length sudden transmission and sleeps during the two sudden transmissions to reduce spare detection.

Noncompetitive MAC protocols are generally used for cluster-based networks. The cluster head is responsible for allocating time slots to all of the sensor nodes within a cluster, collecting and processing data which is sent by the sensor nodes within a cluster, and forwarding data to the sink node.

In this project, we use a MAC mechanism, which is a combination of both competitive and noncompetitive methodologies, which is common practice in WSN technologies. One example of this is within ZigBee, with its purpose illustrated in Figure 3, allowing accommodation of the varied application requirements of a different network number of nodes and different real-time requirements.

Although the most common WSNs are for lowbandwidth applications, the development of technology and the demands of more diversified sensing technologies and more various information acquisition-especially for the application of acquisition of large amounts of data such as the vibration and image data - and the bandwidth requirements for sensor networks have enlarged and the management models required have become more strict.

2.3. Synchronization. In structural health monitoring, there are many requirements regarding synchronous and realtime data acquisition of the vibration information, which are distributed over different parts of an installation. It is especially important for the vibration model analysis of bridge structures, structural stability, and life assessments, which contain a large number of sensor nodes that are distributed over different positions, with different topology structures. The signals must be sampled synchronously by the nodes; otherwise there will be incorrect information (due to samples grouped together coming from different times) of the vibration phase, resulting in an incorrect vibration model judgement. In applications in which sampling frequencies usually exceed $1 \mathrm{KHz}$, the delay of the sensor nodes synchronisation is usually required to be less than $1 \mu \mathrm{s}$. This results in a higher requirement of synchronisation in an SHM WSN.

2.4. Operation Frequency. The wireless carrier frequency has a direct impact on the physical layer transmission accessibility of a network, which plays a significant role in the application of structural health monitoring. Carriers of $2.4 \mathrm{GHz}$ and $868 \mathrm{MHz}$ and $915 \mathrm{MHz}$ are used according to IEEE 802.15.4 and ZigBee Alliance standards listed in Table 1 . The penetration ability through buildings at these frequencies is acceptable, but the diffraction characteristics are relatively poor. When the interior structure of a building is under test, the performance using ZigBee is poor due to poor penetration. In contrast, the $433 \mathrm{MHz}$ ISM band, which is used widely in fields such as automated meter reading (AMR), has achieved relatively good results. In addition, the frequency band of $470 \mathrm{MHz}$ has been selected to be the instrumentation and sensor network special frequency band in China.

Frequencies used in applications such as AMR can be a reference for comparison, classification, and standardisation of the carrier frequency of WSN in SHM.

2.5. Topology and Data Fusion. WSNs need different topologies to meet the needs of different application characteristics in SHM. Typical topologies include star, cluster tree, and mesh networks [9]. Taking the ZigBee network for example, shown in Figure 4, the node is composed of both a reduced function device (RFD) and a full function device (FFD). The ZigBee standard supports the three kinds of topologies that were stated above $[10,11]$.

In the FP7 project mentioned previously, "Health Monitoring of Offshore Wind Farms," the project team compared the characteristics of three different topologies, as we can see in Table 2.

In our practical application, the star network is used for deformation monitoring of low-speed, nontimely applications. In addition to the above three forms, a chain structure (a simplification of mesh) and a combination of the chain structure and the three previous mentioned methodologies has become a commonly used topology.

2.6. Energy Issues. A lot of WSN nodes in SHM applications work in environments where a direct power supply is not available, such as with nodes located upon spinning wind power blades. Therefore, any enhancement of a single node lifetime through the use of low-power technology is a major 


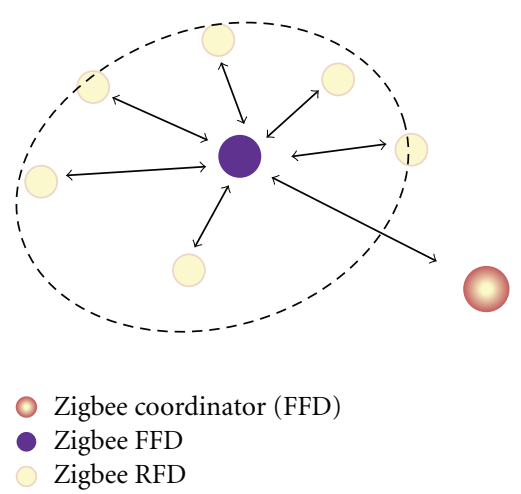

(a)

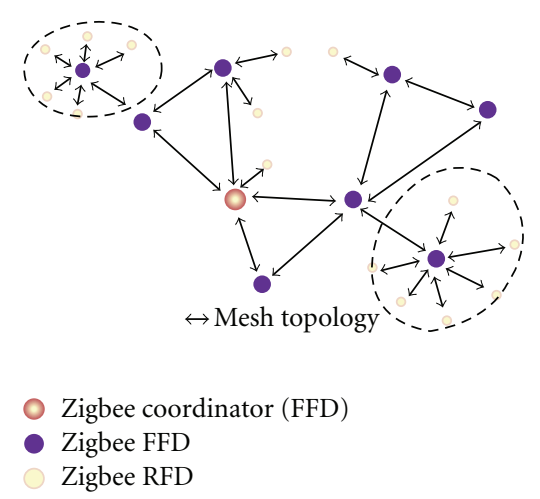

(b)

Figure 4: ZigBee (a) STAR and (b) MESH network topologies.

TABLE 2: Comparison of three kinds of topology for WSN.

\begin{tabular}{|c|c|c|c|}
\hline & Networking features & Advantage & Disadvantage \\
\hline Star & $\begin{array}{l}\text { Supporting point-to-multipoint and } \\
\text { multipoint-to-point communication. } \\
\text { Need for a concentrator or hub } \\
\text { (coordinator for ZigBee network). All } \\
\text { data are transited by hub. } \\
\text { Suited for networking in which the } \\
\text { distance between equipments is small and } \\
\text { scattered in a circular fashion. } \\
\text { Suitable for massive, low-speed, and } \\
\text { non-real-time applications, such as } \\
\text { distortion monitoring. }\end{array}$ & $\begin{array}{l}\text { Brief structure. Protocol is simple by } \\
\text { using a polling mode. }\end{array}$ & $\begin{array}{l}\text { The entire spatial span of the } \\
\text { transmission network is equal to the span } \\
\text { of the physical layer. } \\
\text { Concentrator can carry out the role of } \\
\text { data fusion. Its reliability has the decisive } \\
\text { effect regarding the performance of the } \\
\text { entire network. }\end{array}$ \\
\hline Cluster tree & $\begin{array}{l}\text { Network is composed of many star } \\
\text { topologies, using a star to connect the } \\
\text { concentrator. } \\
\text { Suitable for situations where data needs } \\
\text { to be converged, such as in a structure } \\
\text { monitoring network that has an } \\
\text { intelligent analysis ability. }\end{array}$ & $\begin{array}{l}\text { A leveled and clear structure. } \\
\text { Transmission distance is greater than } \\
\text { the star topology. } \\
\text { Protocol is relatively simple and clearer } \\
\text { than with mesh networks. }\end{array}$ & $\begin{array}{l}\text { All the root nodes of the cluster tree } \\
\text { (concentrator or the convergent point) } \\
\text { are essential nodes, upon which there is } \\
\text { also the demand of a high ability in terms } \\
\text { of data fusion. Energy consumption and } \\
\text { reliability as well as the influence on the } \\
\text { entire network are a major issue. }\end{array}$ \\
\hline Mesh & $\begin{array}{l}\text { All entities can communicate with each } \\
\text { other as long as they are within the scope } \\
\text { of the network and if there is no direct } \\
\text { path; they also can communicate in } \\
\text { "multihop" fashion. } \\
\text { They can form highly complex and large } \\
\text { capacity networks. The network can cover } \\
\text { a large physical space so it is suitable for } \\
\text { installation on scattered structures. } \\
\text { The network also has self-organisation } \\
\text { and self-healing capabilities. }\end{array}$ & $\begin{array}{l}\text { The behaviour of the single node is } \\
\text { relatively consistent. } \\
\text { The capacity of the network is large. } \\
\text { Generally it does not have any essential } \\
\text { node. There are many available } \\
\text { communication routes and the success } \\
\text { ratio of the transmission is increased } \\
\text { in comparison to those gained with } \\
\text { other networks. }\end{array}$ & $\begin{array}{l}\text { The behaviour of the entire network is } \\
\text { not easily controlled. } \\
\text { With an increasing number of nodes, the } \\
\text { complexity of the network behaviour } \\
\text { increases greatly and the efficiency is } \\
\text { reduced. }\end{array}$ \\
\hline
\end{tabular}

issue in the application of WSN. Impacting factors upon the lifetime of nodes and the entire network include the following.

(1) The Working Principle and Mode of Sensing System. Each of the many sensors that adopt different physical principles to detect the different phenomena of strain, vibration, obliquity, cracks, the sampling frequency, working mode, and working energy consumption has a very different working principle. A resistance strain sensor operational current needs range from about $200 \sim 400 \mathrm{uA}$, while pulsed eddy current sensors require an excitation current of up to $1 \mathrm{~A}$ or more. Low-speed, low real-time detection usually operates in a larger working cycle, and vibration signals are usually sampled by continuous measurements. The working mode of each sensing system has a decisive influence on the energy consumption of the overall WSN.

(2) The Low-Power and Dormancy Wake-Up Mechanism of Node. Each node employs its own technology to achieve 
low power consumption, whilst ensuring the monitoring application. This has to be achieved via the work/sleep mode along with a low duty cycle [12]. Currently the dormancy mode of low-power nodes has reduced current consumption to $1 \mathrm{uA}$. Generally, the ZigBee node designed for low-speed and real-time applications can run for a period of 6 months to 2 years, with the power supplied by a single AAA battery. In SHM applications such as strain testing, the duty cycle is usually low and so the life of nodes is long.

(3) The Impact of Network Protocols and Calculated Capacity. The calculated capacity requirements of hardware and for the node itself will have a direct impact upon the function of the node. Low-power requirements often conflict with the processing protocol of the WSN, its secure computational ability, and so on. Each function of a WSN, such as selforganisability, adaptability, signal sampling, and information fusion, requires energy consumption. How to ensure the above-mentioned aspects of performance under the condition of low power consumption is always a WSN most crucial issue.

In addition, reasonable network protocols will also play an enhanced role in the reliability of the entire network. For example, in a tree structure, based on the remaining node energy consumption, the key nodes (such as the tree root node and concentrators) are selected in turn. This approach greatly enhances the reliability of the system.

(4) The Self-Powering Capacity of the Node. Currently, with the tendency towards green energy conservation technology development, the use of self-power-generation technology to support nodes has become a research hotspot. Typical self-generation technologies include solar energy technology, vibration and wind-power generation based on the principle of electromagnetic induction, vibration generation based on the principle of the piezoelectric effect, electrostatic collection techniques, and thermoelectric technology based on the temperature effect [13-16]. Amongst these, ZigBee blends the self-generation node into a self-supported system.

In some SHM applications, vibration is the object of measurement as well as the potential power supply source, such as with the vibration of motors and bridges or the spin of blades on a wind farm. In other circumstances there is a large range of solar or temperature change, especially in the west of China, where wind farms are built on a large scale.

\section{Conclusion}

Wireless sensor networks are one of the supporting technologies in structural health monitoring. Through intelligent, self-organising means, they connect sensor nodes, with a variety of different test objects and working principles into a network along with functions of data processing and integration. Structural health monitoring is a convergence area, with a variety of sensor and information processing technologies. Sampling frequencies, operational modalities of different sensors, their respective transmission bandwidth, and realtime monitoring including interference, wireless operation frequency problems, topology, data integration, and energy issues have been laid out as the key components that will need to be considered carefully when designing the wireless sensor network. In this FP7 project HEMOW, "Health Monitoring of the Offshore Wind Farm Monitoring," our international consortium including research teams from China, India, Poland, and the UK will address the above issues in great detail over the next few years.

\section{Acknowledgments}

The research of this paper is supported by FP7 project "HEMOW," the National Science Foundation of China (50907032/E070104), Key Project of Technology of Jiangsu (SBE200900338), and the Ph.D. Programs Foundation of Ministry of Education of China (20093218120019).

\section{References}

[1] http://www.jlhlabs.com/jhill_cs/jhill_thesis.pdf.

[2] Part 15.4: Wireless Medium Access Control (MAC) and Physical Layer (PHY) Specifications for Low-Rate Wireless Personal Area Networks (WPANs), IEEE 802.15.4, 2009.

[3] http://www.ti.com/.

[4] H. Q. Si and T. G. Wang, "Calculation of the unsteady airloads on wind turbine blades under yawed flow," Modern Physics Letters B, vol. 23, no. 3, pp. 493-496, 2009.

[5] S. Yuan, X. Lai, X. Zhao, X. Xu, and L. Zhang, "Distributed structural health monitoring system based on smart wireless sensor and multi-agent technology," Smart Materials and Structures, vol. 15, no. 1, pp. 1-8, 2006.

[6] Fundamental study of Large Size Wind Turbine Aerodynamics, funded by the National Basic Research Program of China; funding: 35 million RMB; project leader: Tongguang Wang, duration: 2007-2011.

[7] J. W. Wilson, G. Y. Tian, and S. Barrans, "Residual magnetic field sensing for stress measurement," Sensors and Actuators A, vol. 135, no. 2, pp. 381-387, 2007.

[8] C. Shen, C. Srisathapornphat, and C. Jaikaeo, "Sensor information networking architecture and applications," IEEE Personal Communications, vol. 8, no. 4, pp. 52-59, 2001.

[9] J. Yick, B. Mukherjee, and D. Ghosal, "Wireless sensor network survey," Computer Networks, vol. 52, no. 12, pp. 2292-2330, 2008.

[10] ZibBee Alliance, ZigBee Specification, ZigBee Document 053474r06 Version 1.0., 2004.

[11] IEEE Standards 802.15.4 TM-2003.Wireless medium access control (MAC) and physical layer (PHY) specifications for low-rate wireless personal area networks (LR-WPANs), 2003.

[12] K. Vijayaraghavan and R. Rajamani, "Active control based energy harvesting for battery-less wireless traffic sensors: theory and experiments," in Proceedings of the American Control Conference (ACC '08), pp. 4579-4584, June 2008.

[13] A. Hande, T. Polk, W. Walker, and D. Bhatia, "Indoor solar energy harvesting for sensor network router nodes," Microprocessors and Microsystems, vol. 31, no. 6, pp. 420-432, 2007.

[14] Y. K. Tan, K. Y. Hoe, and S. K. Panda, "Energy harvesting using piezoelectric igniter for self-powered radio frequency (RF) wireless sensors," in Proceedings of the IEEE International Conference on Industrial Technology (ICIT '06), pp. 1711-1716, Mumbai, India, December 2006. 
[15] L. Mateu, C. Codrea, N. Lucas, M. Pollak, and P. Spies, "Energy harvesting for wireless communication systems using thermogenerators," in Proceedings of the IEEE XXI Conference on Design of Circuits and Integrated Systems (DCIS '07), Barcelona, Spain, November 2006.

[16] M. T. Penella and M. Gasulla, "A review of commercial energy harvesters for autonomous sensors," in Proceedings of the Instrumentation and Measurement Technology Conference (IMTC '07), pp. 1-5, Warsaw, Poland, May 2007. 

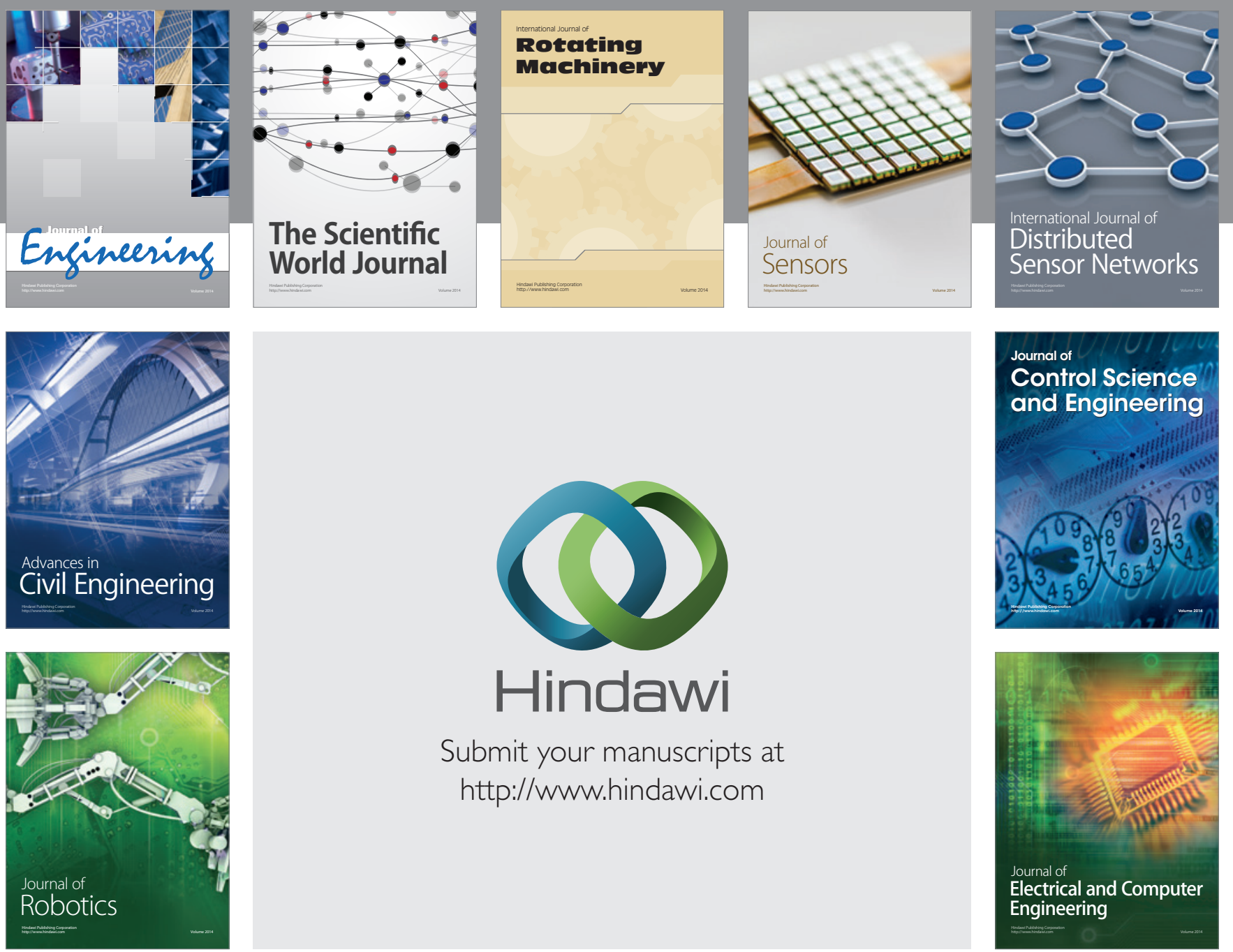

Submit your manuscripts at

http://www.hindawi.com
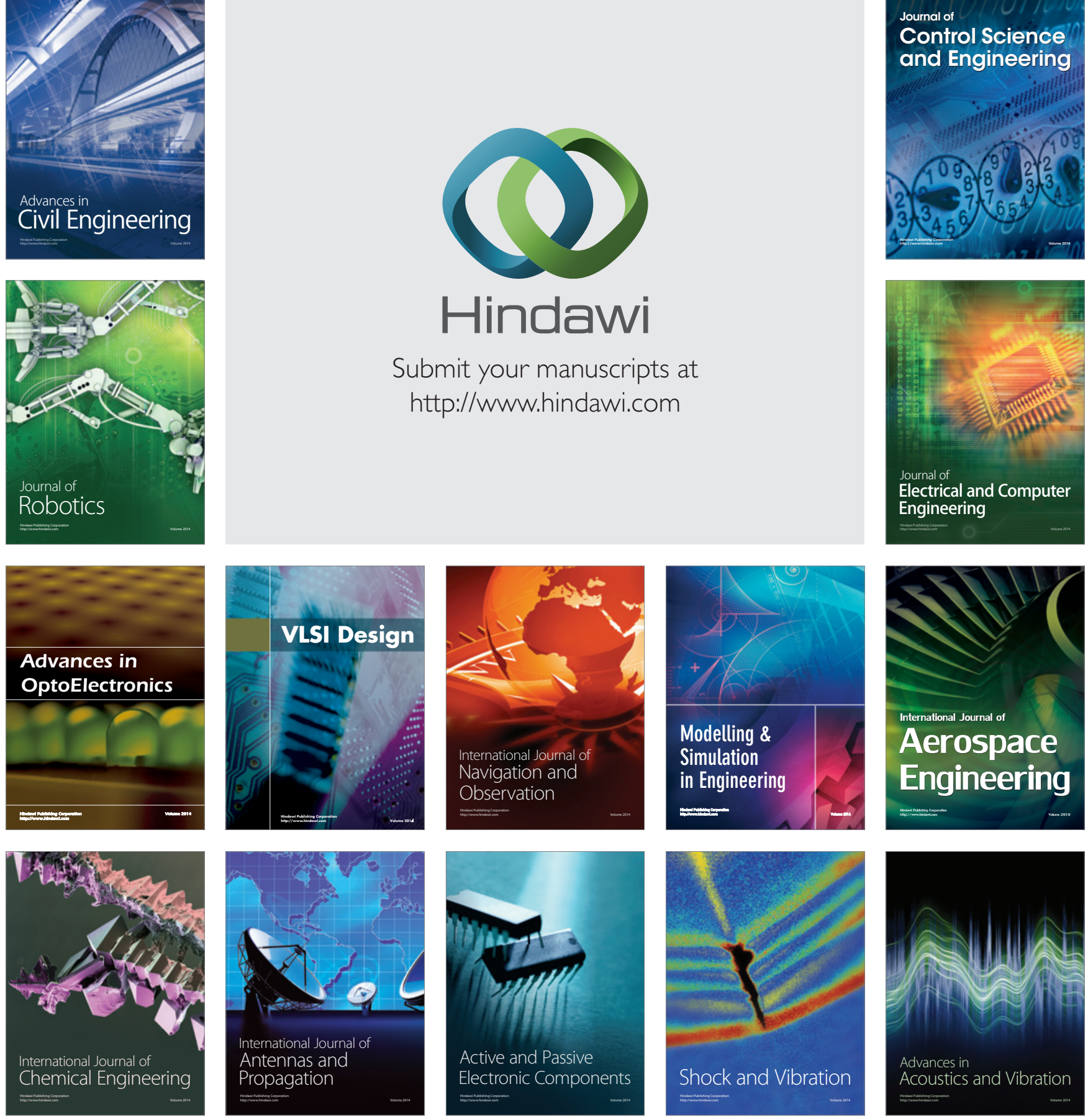